\title{
Epidemiology and outcome of sepsis in a tertiary-care hospital in a developing country
}

\author{
M. D. TANRIOVER*, G. S. GUVEN, D. SEN, S. UNAL AND O. UZUN \\ Hacettepe University School of Medicine, Department of Medicine, Ankara, Turkey
}

(Accepted 1 June 2005, first published online 19 August 2005)

\section{SUMMARY}

Sepsis continues to have a substantial mortality and morbidity despite advances in the diagnosis and management of this condition. We retrospectively analysed hospital charts of patients diagnosed to have sepsis between January 2002 and June 2003. Demographic characteristics of patients, microbiological findings and predictors of survival were evaluated. Sixty-nine sepsis episodes that occurred in 63 patients were analysed. The most common underlying diseases were hypertension, malignancies and diabetes mellitus. Renal insufficiency, respiratory distress and disseminated intravascular coagulation developed in $52 \cdot 2,30 \cdot 4$ and $30.4 \%$ of the episodes respectively; $47.7 \%$ of the blood cultures yielded an organism. Gram-negative bacteria were the predominant microorganisms (65.9\%). Fifty-five patients (87.3\%) died. Mechanical ventilation and underlying renal disease were significant determinants of mortality. In conclusion, Gram-negative bacteria remain the major pathogens in sepsis. The mortality remains very high, and a change in the clinical approach to the septic patient should be employed to improve the outcome.

\section{INTRODUCTION}

Despite the increasing standard of care and supportive therapy, the challenging problem of sepsis is growing [1]. An analysis of more than 10 million cases of sepsis diagnosed between 1979 and 2000 showed an approximately threefold increase in its incidence over the past two decades [2]. More people, including trauma survivors, those with chronic illnesses, in addition to the ageing population, suffer from sepsis. On the other hand, the use of in-dwelling catheters and other invasive procedures creates a portal of entry in this ever-growing population prone to sepsis. As the epidemiology of sepsis changes, definitions also change in an effort to better diagnose, understand the

\footnotetext{
* Author for correspondence: Dr M. D. Tanriover, Hacettepe Universitesi Tıp Fakültesi, Ic Hastaliklari Anabilim Dali, Genel Dahiliye Unitesi, 06100, Sihhiye, Ankara, Turkey.

(Email:mdurusu@hacettepe.edu.tr)
}

pathogenesis, classify and treat this dilemma. The recently introduced PIRO concept (predisposition, insult infection, response, and organ dysfunction) is an effort to integrate the molecular and clinical data to change our understanding of sepsis [3]. Documentation of infection is not always possible or practical; therefore, we need criteria that are both objective and easy to implement at the bedside with a high sensitivity and specificity to diagnose sepsis and systemic inflammatory response syndrome (SIRS).

The spectrum of causative microorganisms has shifted from predominantly Gram-negative bacteria in late 1970s and 1980s to Gram-positive bacteria currently [2]. In addition, fungi have emerged as important pathogens [2]. However, Gram-negative bacteria have gained importance once more especially in the tertiary-care centres $[4,5]$.

There are conflicting data with regard to the incidence and the outcome of sepsis. Studies are often not 
uniform or comparable because definitions of organ dysfunction, classification of patients, timing of events, inclusion criteria and outcome measures vary widely $[6,7]$. One of the major caveats has been the difficulty to standardize the definitions and to distinguish the accompanying disorder from a sepsisrelated dysfunction [8]. The outcome of sepsis is also dependent on whether the study population is severely immunosuppressed, admitted to an intensive care unit (ICU) or managed in a ward, or the study is multicentric or takes place in an ICU of a single hospital.

In this study, we define the characteristics, clinical course, microbiological findings and the outcome of patients diagnosed with sepsis at our centre (Hacettepe University School of Medicine), with emphasis on the factors that are associated with increased mortality.

\section{METHODS}

\section{Study design and variables}

The study was conducted at Hacettepe University Hospital for Adults, an 800-bed tertiary-care centre located in the capital city of Turkey. There are 54 beds in 6 separate ICUs ( 9 internal medicine, 9 surgical, 12 neurosurgical, 12 thoracic-cardiovascular surgical, 4 anaesthesia and reanimation, and 8 coronary).

A retrospective search of hospital charts with a diagnostic code of 'sepsis/septicaemia/bacteraemia' between January 2002 and June 2003 was performed. 'Septic shock' and 'severe sepsis' were included under the code of sepsis. A total of 78 patient charts were identified and 67 were available in the archive. Four of the patients did not fulfil sepsis criteria and were excluded. Sixty-nine sepsis episodes in 63 patients were eligible for analysis.

Sepsis was considered to be present when at least two of the four criteria of SIRS were met $(a)$ body temperature $>38{ }^{\circ} \mathrm{C}$ or $<36{ }^{\circ} \mathrm{C},(b)$ heart rate $>90$ beats/min, (c) respiratory rate $>20 /$ min or partial carbon dioxide pressure $\left(p_{\mathrm{CO} 2}\right)<32 \mathrm{mmHg},(d)$ white blood cell (WBC) count $>12 \times 10^{9} / 1$ or $<4 \times 10^{9} / 1$ or the presence of at least $10 \%$ immature neutrophils, in addition to the presence of microbiologically or clinically documented or suspected infection [9]. Microbiologically documented infection was defined as an infection confirmed by positive cultures of blood or sterile body fluids or a sample obtained from a site of suspected infection, whereas clinically documented infection was defined as the presence of gross purulence or an abscess detected in physical examination, radiographic images and/or histological examination.

Dysfunction of the organ systems were defined as follows. Renal dysfunction: serum creatinine $\geqslant 2.0 \mathrm{mg} / \mathrm{dl}$ or urine output $<0.5 \mathrm{ml} / \mathrm{kg}$ per hour despite adequate fluid resuscitation; hepatic dysfunction: serum bilirubin $>2.0 \mathrm{gr} / \mathrm{dl}$, or a threefold increase in serum aminotransferases; disseminated intravascular coagulation (DIC): international normalized ratio $($ INR) $>1 \cdot 2$, elevated d-dimer, platelet $<100000 /$ $\left.\mathrm{mm}^{3}\right)$; respiratory insufficiency: a $\mathrm{PaO}_{2} / \mathrm{FiO}_{2}$ ratio $\leqslant 200$; hypotension: systolic blood pressure $\leqslant 90 \mathrm{mmHg}$ or the mean arterial blood pressure $\leqslant 70 \mathrm{mmHg}$ despite adequate fluid and vasopressor resuscitation; central nervous system (CNS) dysfunction: acute alteration of the mental status.

During the study period, blood specimens were drawn at the bedside, and cultured in BACTEC medium (Becton, Dickinson \& Company, Clare, Ireland). Subculture to blood agar was performed when the automated alert system signalled growth in any of the bottles, and the agar was then incubated at 5-10\% $\mathrm{CO}_{2}$. Blind subcultures were performed from the bottles with no growth signal on day 7 .

Underlying diseases were classified according to Jackson-McCabe classification [10]. The following data were collected from the hospital charts; age, gender, date of admission to the emergency department (ED)/ICU/wards, date of transfer to the ICU/ wards, date of diagnosis of sepsis, criteria of SIRS, serum biochemical tests, underlying diseases, possible site of infection at the time of sepsis diagnosis, data regarding organ dysfunction, microbiological data, and finally, the need and the duration of mechanical ventilation (MV). Cultures obtained within 3 days before and 5 days after the diagnosis of sepsis were included [11]. Antimicrobials, which were started or continued at the time of sepsis diagnosis, were recorded. The worst value measurements within $24 \mathrm{~h}$ of diagnosis were taken into account. The patients were initially treated with an empirical antimicrobial regimen based on the surveillance data of the hospital infection control committee; antimicrobial therapy was then tailored as required according to the culture results.

The primary outcome measure was death during hospital stay or discharge from the hospital. Six patients had two episodes of sepsis and the second episode was considered separate if at least 3 days had elapsed between the episodes [12]. 
Sepsis was considered to be community-acquired if it was diagnosed within 2 days of hospital admission, after this period it was considered hospital-acquired. The exact time of diagnosis was unclear in one patient who had been referred to our hospital from another centre, and was thus excluded from the analysis of data comparing community- and hospital-acquired episodes.

Antibiotic therapy was considered adequate if at least one antibiotic to which the isolated pathogen was susceptible was administered within $24 \mathrm{~h}$ in adequate doses.

\section{Statistical analysis}

All statistical analyses were performed using SPSS version 10.0 software (SPSS Inc., Chicago, IL, USA). Numerical data were reported as mean \pm standard deviation (S.D.) if normally distributed or median (25th-75th percentile) if not normally distributed, as indicated by the distribution pattern in the onesample Kolmogorov-Smirnov test. To test for the difference between quantitative variables in community- and hospital-acquired sepsis episodes, an independent sample $t$ test was used for variables that are normally distributed. Non-parametric numerical data were analysed with the Mann-Whitney $U$ test. Differences between categorical variables were analysed by $\chi^{2}$ with continuity correction or Fisher's exact test where appropriate. Kaplan-Meier survival analysis was used to define the predictors of mortality. The primary outcome measure was death during hospital stay or discharge from the hospital. This endpoint, expressed in days, was analysed by KaplanMeier survival analysis in a model that used the days of hospital-stay and the underlying diseases, evolving organ dysfunctions and the presence of MV. The survival/hazard functions were compared and contrasted for exposure status to one potential predictor variable at a time (e.g. presence of MV vs. absence). Risk of mortality was studied controlling for the time to event, i.e. the time period (days) between the onset of sepsis and death or recovery/discharge, whichever came first. A $P$ value of $<0.05$ was considered as statistically significant. Crude mortality rates were calculated for 7-, 14-, 28-day and overall mortality.

\section{RESULTS}

Sixty-nine episodes in 63 patients were analysed. Thirty-four of the patients $(54 \%)$ were male; the mean age was $61 \cdot 8 \pm 16 \cdot 5$ years (Table 1 ). Half of the episodes were hospital acquired. The most common underlying diseases were hypertension $(46.4 \%)$, malignancies $(36 \cdot 2 \%)$ and diabetes mellitus $(30 \cdot 4 \%)$. Haematological malignancies constituted $32.0 \%$ of cancer in our patients. According to JacksonMcCabe classification, two patients were in the rapidly fatal group, while 32 patients were in the ultimately fatal and 29 patients were in the non-fatal group. Accompanying chronic obstructive pulmonary disease and renal disease were more common in hospitalacquired episodes than community-acquired episodes ( $\chi^{2}$ test, $P=0.027$ and $P=0.019$ respectively).

Twenty-seven out of $69(39 \cdot 1 \%)$ episodes were diagnosed at the ED; i.e. these patients fulfilled the criteria for the diagnosis of sepsis when they presented at the ED. However, only $40.7 \%$ of the patients could be transferred to an ICU from the ED compared to $85.2 \%$ of those who were diagnosed with sepsis in the medical or surgical wards. In $21.7 \%$ of episodes, sepsis was diagnosed while the patient was in the ICU.

Thirty-nine per cent of the patients were febrile, whereas $8.7 \%$ were hypothermic within $24 \mathrm{~h}$ of sepsis diagnosis. Leukopenia was detected in $21.7 \%$ of the episodes and leukocytosis in 56.5\%. Hypotension was present in $37(53.6 \%)$ episodes. There were no statistical differences between hospital- and community-acquired cases with respect to demographic characteristics and clinical parameters.

A total of 86 blood cultures were evaluated in the study, and $41(47 \cdot 7 \%)$ yielded an organism. No fungal pathogen was isolated from blood cultures. The mean number of blood cultures per episode of sepsis in the previously defined period of time (total 8 days) was $1 \cdot 25$. Of 41 positive blood cultures, 27 $(65.9 \%)$ yielded Gram-negative bacteria. Klebsiella spp. (40.7\%) and Escherichia coli $(29 \cdot 6 \%)$ were the leading pathogens among Gram-negative bacteria. There was a difference in the spectrum of causative pathogens between community- and hospitalacquired episodes. In community-acquired episodes, $16(41 \%)$ out of 39 blood cultures showed bacterial growth, and the majority of pathogens $(56 \cdot 2 \%)$ were Gram-positive organisms. In hospital-acquired episodes, $25(53 \cdot 2 \%)$ out of 47 blood cultures were positive. Strikingly, $80 \%$ of the positive cultures yielded Gram-negative bacteria (Table 2).

Urine samples were cultured in 63 episodes and 14 were positive with $E$. coli being the most common pathogen (42.9\%) followed by Candida spp. in 35.7\% of samples. Pneumonia was the most likely source of 
Table 1. Co-existing diseases, clinical and laboratory variables, sources of infection and evolving organ dysfunctions in subsets of sepsis episodes

\begin{tabular}{|c|c|c|c|c|}
\hline Variable & $\begin{array}{l}\text { All episodes } \\
(n=69)^{*}\end{array}$ & $\begin{array}{l}\text { Community- } \\
\text { acquired } \\
\text { sepsis group } \\
(n=34)\end{array}$ & $\begin{array}{l}\text { Hospital- } \\
\text { acquired sepsis } \\
\text { group } \\
(n=34)\end{array}$ & $P$ value $\dagger$ \\
\hline Age (years) & $61 \cdot 8 \pm 16 \cdot 5$ & $64 \cdot 9 \pm 13 \cdot 8$ & $59 \cdot 0 \pm 18 \cdot 4$ & \\
\hline \multicolumn{5}{|l|}{$\operatorname{Sex}(\%)$} \\
\hline Female & $46 \cdot 0$ & $50 \cdot 0$ & $47 \cdot 1$ & \\
\hline Male & $54 \cdot 0$ & $50 \cdot 0$ & $52 \cdot 9$ & \\
\hline \multicolumn{5}{|l|}{ SIRS criteria } \\
\hline Temperature $\left({ }^{\circ} \mathrm{C}\right)$ & $37 \cdot 5 \pm 1 \cdot 6$ & $37 \cdot 3 \pm 1 \cdot 5$ & $37 \cdot 6 \pm 1 \cdot 8$ & \\
\hline Heart rate (beats/min) & $118 \cdot 4 \pm 27 \cdot 5$ & $121 \cdot 6 \pm 23 \cdot 9$ & $114 \cdot 0 \pm 32 \cdot 0$ & \\
\hline Systolic blood pressure (mmHg) & $83 \cdot 0(70 \cdot 0-93 \cdot 5)$ & $75 \cdot 7 \pm 36 \cdot 7$ & $81 \cdot 1 \pm 26 \cdot 0$ & \\
\hline Diastolic blood pressure (mmHg) & $44 \cdot 5 \pm 20 \cdot 3$ & $43 \cdot 1 \pm 23 \cdot 2$ & $45 \cdot 6 \pm 17 \cdot 4$ & \\
\hline Respiratory rate (breaths/min) & $32 \cdot 2 \pm 8 \cdot 2$ & $33 \cdot 4 \pm 8 \cdot 2$ & $30 \cdot 5 \pm 8 \cdot 2$ & \\
\hline Partial pressure of carbon dioxide $(\mathrm{mmHg})$ & $33 \cdot 3 \pm 12 \cdot 7$ & $33 \cdot 4 \pm 13 \cdot 2$ & $33 \cdot 9 \pm 12 \cdot 3$ & \\
\hline White blood cell count (per $\mathrm{mm}^{3}$ ) & $12900(4100-18250)$ & $12206 \pm 9593$ & $12850(4325-21725)$ & \\
\hline \multicolumn{5}{|l|}{ Laboratory values at the time of diagnosist } \\
\hline Blood urea nitrogen (mg/dl) & $48 \cdot 3 \pm 32 \cdot 1$ & $50 \cdot 7 \pm 36 \cdot 8$ & $45 \cdot 1 \pm 26 \cdot 9$ & \\
\hline Creatinine (mg/dl) & $1 \cdot 6(0 \cdot 9-3 \cdot 0)$ & $2 \cdot 5(0 \cdot 9-3 \cdot 4)$ & $1 \cdot 5(0 \cdot 9-2 \cdot 7)$ & \\
\hline Albumin $(\mathrm{g} / \mathrm{dl})$ & $2 \cdot 5 \pm 0 \cdot 6$ & $2 \cdot 6 \pm 0 \cdot 6$ & $2 \cdot 4 \pm 0 \cdot 6$ & \\
\hline Total bilirubin (mg/dl) & $1 \cdot 5(0 \cdot 8-3 \cdot 7)$ & $1 \cdot 6(0 \cdot 9-4 \cdot 2)$ & $1 \cdot 5(0 \cdot 6-2 \cdot 3)$ & \\
\hline Alanine aminotransferase (U/1) & $35(23-69)$ & $41(24-92)$ & $31(20-603)$ & \\
\hline Gamma-glutamyltransferase (U/1) & $65(34-118)$ & $63(29-120)$ & $88 \cdot 1 \pm 79 \cdot 2$ & \\
\hline Anion gap (mequiv./l) & $21 \cdot 3 \pm 7 \cdot 9$ & $21 \cdot 2 \pm 6 \cdot 9$ & $20 \cdot 7 \pm 8 \cdot 6$ & \\
\hline \multicolumn{5}{|l|}{ Coexisting diseases $(\%)$} \\
\hline Coronary artery disease & $29 \cdot 0$ & $23 \cdot 5$ & $35 \cdot 3$ & \\
\hline Chronic obstructive pulmonary disease & $13 \cdot 0$ & $2 \cdot 9$ & $20 \cdot 6$ & $\mathbf{0} \cdot 027$ \\
\hline Diabetes mellitus & $30 \cdot 4$ & $20 \cdot 6$ & $41 \cdot 2$ & \\
\hline Hypertension & $46 \cdot 4$ & $38 \cdot 2$ & $55 \cdot 9$ & \\
\hline Liver disease & $20 \cdot 3$ & $20 \cdot 6$ & $20 \cdot 6$ & \\
\hline Malignancy & $36 \cdot 2$ & $32 \cdot 4$ & $41 \cdot 2$ & \\
\hline Neurological disease & $23 \cdot 2$ & $32 \cdot 4$ & $14 \cdot 7$ & \\
\hline Renal disease & $21 \cdot 7$ & $8 \cdot 8$ & $35 \cdot 3$ & $0 \cdot 019$ \\
\hline \multicolumn{5}{|l|}{ Diagnosis $(\%)$} \\
\hline Pneumonia & $44 \cdot 9$ & $38 \cdot 2$ & $50 \cdot 0$ & \\
\hline Urosepsis & $13 \cdot 0$ & $11 \cdot 8$ & $14 \cdot 7$ & \\
\hline Peritonitis & $4 \cdot 3$ & $8 \cdot 8$ & $0 \cdot 0$ & \\
\hline Febrile neutropenia & $8 \cdot 7$ & $11 \cdot 7$ & $5 \cdot 9$ & \\
\hline Intra-abdominal surgical process & $23 \cdot 2$ & $23 \cdot 5$ & $20 \cdot 6$ & \\
\hline Skin infections & $10 \cdot 2$ & $5 \cdot 9$ & $14 \cdot 7$ & \\
\hline Soft tissue infections & $11 \cdot 6$ & $11 \cdot 8$ & $14 \cdot 7$ & \\
\hline Bone infections & $4 \cdot 4$ & $0 \cdot 0$ & $5 \cdot 9$ & \\
\hline \multicolumn{5}{|l|}{ Evolving organ dysfunction (\%) } \\
\hline Acute renal failure & $52 \cdot 2$ & $58 \cdot 8$ & $47 \cdot 1$ & \\
\hline Respiratory failure & $30 \cdot 4$ & $23 \cdot 5$ & $35 \cdot 3$ & \\
\hline Haematological process & $30 \cdot 4$ & $23 \cdot 5$ & $38 \cdot 2$ & \\
\hline
\end{tabular}

SIRS, Systemic inflammatory response syndrome.

* Acquisition place of sepsis is unclear in one patient.

$\dagger$ To test for the difference between quantitative variables in community- and hospital-acquired sepsis episodes, independent sample $t$ test is used for variables that are normally distributed. Non-parametric numerical data were analysed with Mann-Whitney $U$ test. Difference between the qualitative variables in two groups is analysed by $\chi^{2}$ with continuity correction or Fisher's exact test where appropriate.

† Worst values within $24 \mathrm{~h}$ of diagnosis of sepsis expressed as means \pm s.D. for the variables that are distributed normally or median (25th-75th percentile) for those distributed non-normally. 
Table 2. Bloodstream infections in community- and hospital-acquired sepsis episodes

\begin{tabular}{|c|c|c|c|}
\hline Pathogen & $\begin{array}{l}\text { Total number of } \\
\text { isolates }(\%)\end{array}$ & $\begin{array}{l}\text { Community-acquired } \\
\text { episodes }\end{array}$ & $\begin{array}{l}\text { Hospital-acquired } \\
\text { episodes }\end{array}$ \\
\hline Gram-positive & $14(34 \cdot 1)$ & $9(56 \cdot 2)$ & $5(20 \cdot 0)$ \\
\hline Staphylococcus aureus & $1(2 \cdot 4)$ & $1(6 \cdot 2)$ & \\
\hline Staphylococcus epidermidis & $5(12 \cdot 2)$ & $3(18 \cdot 8)$ & $2(8 \cdot 0)$ \\
\hline Staphylococcus haemolyticus & $2(4 \cdot 9)$ & & $2(8 \cdot 0)$ \\
\hline Staphylococcus capitis & $1(2 \cdot 4)$ & $1(6 \cdot 2)$ & \\
\hline Streptococcus pneumoniae & $1(2 \cdot 4)$ & & $1(4 \cdot 0)$ \\
\hline Group A $\beta$-haemolytic streptococcus* & $4(9 \cdot 8)$ & $4(25 \cdot 0)$ & \\
\hline Gram-negative & $27(65 \cdot 9)$ & $7(43 \cdot 8)$ & $20(80 \cdot 0)$ \\
\hline Escherichia coli & $8(19 \cdot 5)$ & $1(6 \cdot 3)$ & $7(28 \cdot 0)$ \\
\hline Klebsiella pneumoniae & $8(19 \cdot 5)$ & $2(12 \cdot 5)$ & $6(24 \cdot 0)$ \\
\hline Klebsiella oxytoca & $3(7 \cdot 3)$ & & $3(12 \cdot 0)$ \\
\hline Acinetobacter lwoffii & $2(4 \cdot 9)$ & $2(12 \cdot 5)$ & \\
\hline Acinetobacter baumannii & $1(2 \cdot 4)$ & & $1(4 \cdot 0)$ \\
\hline Serratia marcescens & $1(2 \cdot 4)$ & & $1(4 \cdot 0)$ \\
\hline Serratia odorifera & $2(4 \cdot 9)$ & & $2(8 \cdot 0)$ \\
\hline Enterobacter gergoviae & $2(4 \cdot 9)$ & $2(12 \cdot 5)$ & \\
\hline
\end{tabular}

* Isolates from a single patient.

infection, as recognized in $44.9 \%$ of sepsis episodes at the time of diagnosis. MV was required in $52(75.4 \%)$ of the episodes. A similar number of patients with community-acquired sepsis required MV compared to those with hospital-acquired infection $(70.6 \% \mathrm{vs}$. $79.4 \%, P=0.575)$. The median duration of $\mathrm{MV}$ was 4 days (25th and 75th percentiles: 1 and $11 \cdot 3$ days respectively). Among 16 patients admitted with an abdominal surgical pathology, biliary tract disorders were detected in $62.5 \%$. Skin, soft tissue and bone infections were the remaining sources of infection in $10 \cdot 2,11 \cdot 6$ and $4 \cdot 4 \%$ of episodes respectively.

The most common organ dysfunction that developed during sepsis was renal insufficiency $(52 \cdot 2 \%)$, followed by respiratory failure $(30.4 \%)$ and DIC $(30 \cdot 4 \%)$. There was no difference between community- and hospital-acquired episodes with regard to the development of organ dysfunction.

In $17(24 \cdot 6 \%)$ of the episodes, initial antibiotic monotherapy (imipenem or meropenem in seven episodes, piperacillin-tazobactam and ampicillinsulbactam each in three episodes, ceftriaxone in two, vancomycin and levofloxacin each in one episode) was preferred over combination therapy. The most common combination administered was ampicillinsulbactam plus ciprofloxacin in nine $(13 \cdot 1 \%)$ episodes, followed by imipenem/meropenem plus a glycopeptide and piperacillin-tazobactam plus a glycopeptide, each in six $(8.7 \%)$ episodes. Thirty-three episodes could be evaluated for the adequacy of antimicrobial therapy. Twenty-six $(78 \cdot 8 \%)$ of the antibiotic regimens were adequate, whereas seven $(21.2 \%)$ were not.

Fifty-five of the 63 patients $(87 \cdot 3 \%)$ died during the hospital stay. The mortality rate was $92 \cdot 2 \%$ when at least one organ dysfunction (respiratory, renal, hepatic or haematological) was present, compared to $44.4 \%$ if none was present. The crude mortality of the patients diagnosed in the ED, ICU and wards was $69 \cdot 6,92 \cdot 9$ and $96 \cdot 2 \%$ respectively. In those episodes treated with adequate antimicrobial therapy, the mortality was $76.9 \%$, compared to $100 \%$ in patients treated inadequately. Among non-survivors, the median length of stay in the hospital from the time of admission was 13 days (range 134 days) and it was 5 days (range 44 days) from the time of diagnosis. Seven-, 14- and 28-day mortality rates were $52 \cdot 2,63 \cdot 8$ and $76.8 \%$ respectively. Kaplan-Meier analyses, using the number of days from diagnosis until death or discharge from the hospital as outcome measure, revealed that MV and underlying renal disease were significant determinants of mortality $(P=0.0004$ and $P=0.0023$ respectively).

Patients with community-acquired sepsis had a crude mortality of $67.6 \%$ during the study period, and 7-, 14- and 28-day mortalities were 50, 58.8 and $67 \cdot 7 \%$ respectively (Fig.). The outcome was much poorer in hospital-acquired episodes with a crude mortality of $91 \cdot 2 \%$ and $7-, 14-$ and 28-day mortalities of $52 \cdot 9,67 \cdot 7$ and $85 \cdot 3 \%$ respectively. 


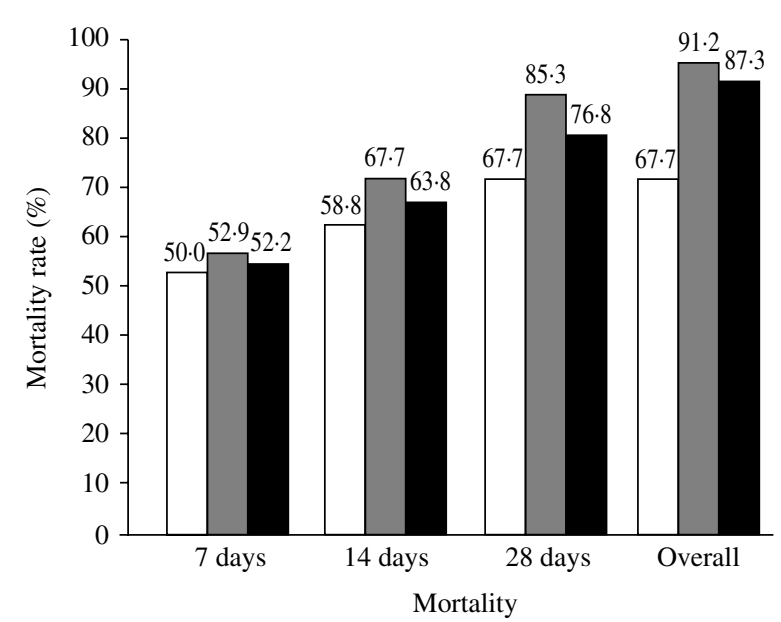

Fig. Mortality rates in community-acquired ( $\square)$; hospitalacquired ( $\square$ ) and all ( $\square$ ) sepsis episodes.

\section{DISCUSSION}

These findings, although limited by the retrospective nature of the study, reflect a strikingly high mortality in a tertiary-care centre in Turkey. Sepsis is a syndrome with grave consequences and septic patients should be monitored in an ICU. However, we could transfer only $40 \cdot 7 \%$ of the septic patients to the ICU from the ED due to the fact that an intermediate care unit is lacking at our centre, leading to a slow turnover of the ICU population. The limited numbers of ICU beds are usually occupied with long-standing patients who have chronic diseases and sometimes irreversible organ damage, and this precludes the timely transfer of patients who need to be admitted to the ICU.

In an era of revisited definitions, microbiological documentation is no more a 'must' to make the diagnosis of sepsis. The yield of blood cultures is low, as demonstrated by our finding $(47 \cdot 7 \%)$, as well as by others [12-14]. In our series, Gram-negative bacilli constituted the majority of pathogens $(65.9 \%)$ isolated from bacteraemic patients in the hospital setting. Although the general trend is an increase in Gram-positive bacteraemic episodes in the literature, some studies show a high incidence of Gram-negative bacteria in bloodstream infections $[4,5,15]$. This is observed especially in hospitals where antimicrobial resistance is high and patients are managed with broad-spectrum antibiotics $[14,16,17]$. In a review by Brun-Buisson, Gram-negative bacteria predominated in severe sepsis episodes [6].

We observed some changes in the spectrum of pathogens at our centre. Uzun et al. reported that
E. coli $(34 \cdot 7 \%)$ and Enterobacter spp. (32.2\%) were the most common pathogens in 448 episodes of bacteraemia caused by Gram-negative bacilli between 1983 and 1989 [18]. Only $2 \cdot 3 \%$ of the episodes were caused by Klebsiella spp. Our present data demonstrate a similar frequency for E. coli $(37.5 \%)$, however, Klebsiella spp. (43.8\%) have become the most common pathogens in Gram-negative bacteraemic episodes. Although the study populations in these two studies are different, the dramatic increase in the incidence of Klebsiella spp. is worth mentioning. This might be explained by the improvement in bacterial identification techniques over the years, since most of those Enterobacter spp. were later reidentified as Klebsiella (O. Uzun, unpublished data).

Because the study is retrospective, we could not demonstrate the antimicrobial resistance rates and expression rate of extended-spectrum $\beta$-lactamases (ESBLs) in the strains reported. However, the database of the hospital infection control committee revealed that in 2003 the overall resistance of Acinetobacter spp. to imipenem was $63 \%$ and to ceftazidime was $85 \%$. Almost all E. coli were susceptible to imipenem $(98 \%)$, but displayed a resistance rate of $31 \%$ to ceftazidime. The susceptibilities of Klebsiella spp. to imipenem were between $93 \%$ and $96 \%$, whereas in the case of ceftazidime, resistance rates of $21-23 \%$ were observed. Since resistance to ceftazidime mirrors the expression of ESBLs, onethird of E. coli strains and one-fifth of Klebsiella strains may have been ESBL producers (Y. CetinkayaSardan, unpublished data).

Renal failure was the most common organ dysfunction that developed during the course of sepsis in our patients. This is important because renal failure has been associated with a particularly poor prognosis $[7,19]$. In our study, underlying renal disease was also a prognostic factor, as shown by Gogos et al. [16]. It could be hypothesized that, in the acute phase, deterioration in renal function results in hypervolaemia, pulmonary oedema, and decreased clearance of some antimicrobials and other drugs that make the readily stressed patient even harder to handle. In addition, the pharmacokinetics of the antibiotics change, which might lead to sub-therapeutic levels for the antimicrobials for which serum levels are not monitored. The long-term impact of renal failure is the possibility of the need of lifelong renal replacement therapy, which is a subject of discussion regarding the quality of life and long-term survival of septic patients who survive the acute event. 
The exceptionally high mortality in our hospital is worth discussing. To our knowledge, this is the first study that investigates the outcome of sepsis in patients with diverse underlying problems in a tertiarycare hospital in Turkey. Previous studies have defined the epidemiology of certain pathogens or of sepsis in certain group of patients such as neonates, burn patients, trauma or cancer patients. A review of the Turkish Medline and international literature did not yield any similar studies that are comparable with ours. The results of this study may not reflect the trends in other regions of Turkey, or in other countries with different socioeconomic conditions and developmental levels and might, therefore, be regarded as the experience of a single centre.

Twenty-eight-day mortality rates have been reported between $28 \%$ and $65 \%$ in severe sepsis or septic shock in different series $[6,7,11,13,14,19]$. University-affiliated hospitals reported higher mortality rates in previous studies [7]. Although the study designs are not uniform, data from different parts of the world also reveal a considerable range of mortality rate. One Australian study demonstrated a $31 \cdot 1 \%$ mortality rate, whereas a French registry revealed a 2 -month mortality rate of $42 \%$ in severe sepsis $[20,21]$. On the other hand, the mortality of severe sepsis was reported to be $55 \%$ by a Polish group [22], and $54 \%$ mortality in severe sepsis and septic shock was reported in a Russian study [23].

Shock was found to be the only independent factor increasing mortality by Topeli et al. in a series of bacteraemia caused by coagulase-negative staphylococci in our centre [24]. Another study from our centre showed that, the crude mortality rate of patients with Gram-negative bacteraemia increased from $23.3 \%$ to $74 \cdot 3 \%$ in the presence of accompanying shock, and/or organ failure [18]. In our series, the presence of one or more of the organ dysfunctions increased mortality from $44.4 \%$ to $92.2 \%$. The patients diagnosed at the ED had the lowest mortality among all, probably due to the fact that these were community-acquired episodes. On the other hand, mortality approached $100 \%$ for the cases diagnosed in the wards.

In a study by Lundberg et al., the mortality rates for patients who developed shock in wards and ICUs were found to be $70 \%$ and $39 \%$ respectively [11]. We found no significant difference between the outcomes of patients who developed sepsis in the wards or ICUs (92.9\% and $96.2 \%$ respectively). This might be explained by the frequent use of MV in our series
(75.4\%). Unfortunately, Lundberg's study does not provide data on the rate of MV in their population. Pittet et al. also found MV to be an independent predictor of mortality in septic patients [25]. The significant difference in terms of underlying renal failure and chronic obstructive pulmonary disease (although there was no difference in MV frequencies) between the community- and hospital-acquired episodes may also contribute to the especially high mortality in the hospital-acquired episodes.

Although the study design is different-i.e. septic patients in the present series $v s$. bacteraemic patients in the previous reports $[18,24]$-there appears to be no improvement in the prognosis of patients in our centre in the last decade. This mandates a meticulous search for the causes of such a high mortality and implementation of corrective measures in our hospital, although this may not be applicable to other institutions. The present study does not provide specific answers; however, several potential flaws in our daily care of septic patients could be listed, such as a low rate and a delay of transfer of the patients with sepsis to an ICU; failure to monitor the patient adequately and delay and/or lack of appropriate administration of fluid and vasopressor therapy in ED or wards as soon as sepsis is diagnosed.

In conclusion, sepsis continues to carry a high mortality rate especially when it is associated with shock and organ dysfunctions. MV is an independent determinant of mortality. Gram-negative microorganisms such as Klebsiella spp. and E. coli play a major role, particularly in hospitalized patients. However, a delay or failure to transfer the patient to an ICU in a timely manner might be the most important prognostic factor.

\section{DECLARATION OF INTEREST}

None.

\section{REFERENCES}

1. Riedemann NC, Guo RF, Ward PA. The enigma of sepsis. J Clin Invest 2003; 112: 460-467.

2. Martin GS, Mannino DM, Eaton S, Moss M. The epidemiology of sepsis in the United States from 1979 through 2000. N Engl J Med 2003; 348: 1546-1554.

3. Levy MM, Fink MP, Marshall JC, et al. SCCM/ ESICM/ACCP/ATS/SIS. 2001 SCCM/ESICM/ACCP/ ATS/SIS International Sepsis Definitions Conference. Crit Care Med 2003; 31 : 1250-1256. 
4. Arpi M, Renneberg J, Andersen HK, Nielsen B, Larsen SO. Bacteremia at a Danish university hospital during a twenty-five-year period (1968-1992). Scand J Infect Dis 1995; 27: 245-251.

5. Velasco E, Byington R, Martins CSA, Schirmer M, Dias LCM, Gonçalves VMSC. Bloodstream infection surveillance in a cancer centre: a prospective look at clinical microbiology aspects. Clin Microbiol Infect 2004; 10: 542-549.

6. Brun-Buisson C. The epidemiology of the systemic inflammatory response. Intensive Care Med 2000; 26 (Suppl 1): S64-S74.

7. Padkin A, Goldfrad C, Brady AR, Young D, Black N, Rowan K. Epidemiology of severe sepsis occurring in the first 24 hrs in intensive care units in England, Wales, and Northern Ireland. Crit Care Med 2003; 31: 2332-2338.

8. Opal SM. Severe sepsis and septic shock: defining the clinical problem. Scand J Infect Dis 2003; 35: 529-534.

9. Garner JS, Jarvis WR, Emori TG, Horan TC, Hughes JM. CDC definitions for nosocomial infections, 1988. Am J Infect Control 1988; 16: 128-140.

10. McCabe WR, Jackson GG. Gram-negative bacteremia: I. Etiology and ecology. Arch Intern Med 1962; 110: 847-855.

11. Lundberg JS, Perl TM, Wiblin T, et al. Septic shock: an analysis of outcomes for patients with onset on hospital wards versus intensive care units. Crit Care Med 1988; 26: 1020-1024.

12. Sands KE, Bates DW, Lanken PN, et al. Epidemiology of sepsis syndrome in 8 academic medical centers. Academic Medical Center Consortium Sepsis Project Working Group. J Am Med Assoc 1997; 278: 234-240.

13. Harbarth S, Garbino J, Pugin J, Romand JA, Lew D, Pittet D. Inappropriate initial antimicrobial therapy and its effect on survival in a clinical trial of immunomodulating therapy for severe sepsis. Am J Med 2003; 115: 529-535.

14. Garnacho-Montero J, Garcia-Garmendia JL, BarreroAlmodovar A, Jimenez-Jimenez FJ, Perez-Paredes C, Ortiz-Leyba C. Impact of adequate empirical antibiotic therapy on the outcome of patients admitted to the intensive care unit with sepsis. Crit Care Med 2003; 31 : 2742-2751.

15. Vazquez F, Mendoza MC, Villar MH, Perez F, Mendez FJ. Survey of bacteraemia in a Spanish hospital over a decade (1981-1990). J Hosp Infect 1994; 26: 111-121.

16. Gogos CA, Lekkou A, Papageorgiou O, Siagris D, Skoutelis A, Bassaris HP. Clinical prognostic markers in patients with severe sepsis: a prospective analysis of 139 consecutive cases. J Infect 2003; 47: 300-306.

17. Alberti C, Brun-Buisson $\mathbf{C}$, Burchardi $\mathbf{H}$, et al. Epidemiology of sepsis and infection in ICU patients from an international multicentre cohort study. Intensive Care Med 2002; 28: 108-121.

18. Uzun Ö, Akalin HE, Hayran M, Ünal S. Factors influencing prognosis in bacteremia due to Gram-negative organisms: evaluation of 448 episodes in a Turkish University Hospital. Clin Infect Dis 1992; 15: 866-873.

19. Clermont G, Angus DC, Kalassian KG, et al. Reassessing the value of short-term mortality in sepsis: comparing conventional approaches to modeling. Crit Care Med 2003; 31: 2627-2633.

20. Sundararjan V, MacIsaac C, Presneill J, Cade JF, Visvanathan K. Epidemiology of sepsis in Victoria, Australia. Crit Care Med 2005; 33: 71-80.

21. Brun-Buisson C, Meshaka P, Pinton P, Vallet B, EPISEPSIS Study Group. EPISEPSIS : a reappraisal of the epidemiology and outcome of severe sepsis in French intensive care units. Intensive Care Med 2004; 30: 580-588.

22. Kubler A, Durek G, Zamirowska A, et al. Severe sepsis in Poland: results of internet surveillance of 1043 cases. Med Sci Monit 2004; 10: 635-641.

23. Busund R, Koukline V, Utrobin U, Nedashkovsky E. Plasmapheresis in severe sepsis and septic shock: a prospective, randomised, controlled trial. Intensive Care Med 2002; 28: 1434-1439.

24. Topeli A, Ünal S, Hayran M, Akalın HE. Septic shock as a predictor of mortality in bacteremia caused by coagulase-negative staphylococci. Eur J Clin Microbiol Infect Dis 1997; 16: 411-416.

25. Pittet D, Thievent B, Wenzel RP, Li N, Auckenthaler R, Suter PM. Bedside prediction of mortality from bacteremic sepsis. A dynamic analysis of ICU patients. Am J Respir Crit Care Med 1996; 153: 684-693. 